\title{
Lemierre's syndrome complicated by cerebral venous sinus thrombosis: A life threatening and rare disease successfully treated with empiric antimicrobial therapy and conservative approach
}

\author{
Maurizio Giorelli ${ }^{1, *}$, Sergio Altomare ${ }^{1}$, Maria Stella Aniello ${ }^{1}$, Ruggiero Leone ${ }^{1}$, \\ Daniele Liuzzi ${ }^{1}$, Immacolata Plasmati ${ }^{1}$, Michele Sardaro ${ }^{1}$, Maria Superbo ${ }^{1}$, \\ Giuseppe Mennea ${ }^{2}$, Nicola Fioretto ${ }^{3}$, Giuseppe Guglielmi ${ }^{3}$, Rosario Balzano ${ }^{3}$, \\ Tommaso Scarabino ${ }^{4}$, Giuseppe Cuccorese ${ }^{5}$, Francesca Cialdella ${ }^{6}$, \\ Giuseppe Campobasso ${ }^{6}$, Michele Barbara ${ }^{6}$
}

${ }^{1}$ Operative Unit of Neurology, "Dimiccoli" General Hospital, Barletta, Italy;

${ }^{2}$ Operative Unit of Internal Medicine, "Bonomo" General Hospital, Andria, Italy;

${ }^{3}$ Operative Unit of Radiology, "Dimiccoli" General Hospital, Barletta, Italy;

${ }^{4}$ Operative Unit of Radiology, "Bonomo" General Hospital, Andria, Italy;

${ }^{5}$ Operative Unit of Internal Medicine, "Dimiccoli" General Hospital, Barletta, Italy;

${ }^{6}$ Operative Unit of Otorhinolaryngology, "Dimiccoli" General Hospital, Barletta, Italy.

SUMMARY Lemierre's syndrome (LS) is a "forgotten" condition characterized by septic thrombophlebitis of the jugular vein that follows an otolaryngological infection. Fusobacterium necrophorum is the aetiological agent responsible for the syndrome in adolescents and young adults whereas in older people even common bacteria are involved. Complications arise from spreading of septic emboli distally, i.e. to the brain, lungs, bones and internal organs everywhere in the body. We report a middle-aged woman who presented with headache and bilateral sixth cranial nerve palsy following a sphenoidal sinusitis and left mastoiditis. Imaging revealed thrombotic involvement of the left internal jugular vein as well as of several cerebral venous sinuses thrombosis (CVT). Currently, precise management protocols of LS with CVT complication do not exist although a combination of macrolides and second or third-generation cephalosporins, as well as anti-coagulants represent the mainstream of therapeutics. Surgical drainage is associated to remove septic foci but is burdened by severe complications and side effects. Complete recovery was achieved following pharmacological treatment in our patient. This report adds further evidence that LS complicated by CVT may be effectively treated adopting a conservative approach thus avoiding surgical drainage and severe complications.

Keywords jugular vein thrombosis, cerebral venous circulation, sinusitis, otomastoiditis

Lemierre's syndrome (LS) is a rare and potentially life-threatening condition that follows oropharyngeal infection. It usually occurs in adolescents and young adults and is mostly associated with infection of upper airways (1-3). Infection triggers a septic thrombophlebitis of the jugular vein, which can spread to cerebral sinuses, lungs, liver, spleen, joints, and heart (3). In the preantibiotic era, LS had a case-mortality rate ranging from $32 \%$ to $90 \%$ (4) decreased currently to $17 \%$ despite best medical practice $(1,2)$.

A 68-year-old woman with a 15-days history of fever, frontal headache and vomiting presented to ED. She reported intermittent fever, binocular diplopia in the left direction of gaze and xerostomia. CT brain/ neck angiography revealed occlusion of the left internal jugular vein at its origin and its main secondary branches. Thrombosis of the sigmoid cerebral sinus was also apparent (Figure 1). Based on these findings, the patient was admitted to the Neurological unit. Neurological examination revealed only left VI cranial nerve palsy while physical examination showed herpes labialis and a non-painful, tense-elastic, swelling at the left retromandibular level.

Laboratory tests revealed increased d-dimers $(2,501$ $\mathrm{ng} / \mathrm{mL})$, leukocytes (14,890 WBC/ $\mu \mathrm{L}, 89 \%$ neutrophils), and C-reactive protein (CPR) (31.3 mg/dL). Serology 
for common viruses and bacteria was negative as well as blood cultures, onconeural paraneoplastic antibodies, anti-gangliosides, tumor markers and antiphospholipids.

In suspect of Lemierre's syndrome, empirical antibiotic therapy was started. Therapy included Enoxaparin 6,000 IU b.i.d s.c., Ceftriaxone $2 \mathrm{~g}$ b.i.d i.v., Linezolid $600 \mathrm{mg}$ b.i.d., and Acyclovir $5 \mathrm{mg} / \mathrm{kg} / \mathrm{die}$ t.i.d. Starting from the fifth day from admission, the patient was afebrile and gradually we observed normalization of WBC counts $\left(6.09 \times 10^{3}\right.$ cells $\left./ \mu \mathrm{L}\right)$ and reduction of CRP $(5.61 \mathrm{mg} / \mathrm{dL})$.

At the ninth day, head and neck MRI revealed a solid mass $(2.7 \times 1.7 \mathrm{~cm})$ in the context of the left parotid gland, thrombosis of the left transverse sinus, the left sigmoid sinus, the origin of the left internal jugular internal vein and the deep facial venous plexus of the same side. Partial thrombosis was even detected in the right transverse sinus. The sphenoidal sinus was obliterated by fluid material and showed parietal thickening. Some left mastoidal cells resulted in obliteration by fluid material similarly. MRI revealed also inflammatory involvement of the interstitial tissue surrounding the left jugular vein with spreading towards the upper airways (Figure 2). Subsequently, by fine needle aspiration, cytological examination of the parotidal mass was performed, revealing the presence of adenomatous cells.

Head and neck MRI performed 10 days later revealed partial thrombosis of the origin of the left internal jugular vein with inflammatory involvement of surrounding soft tissues, sphenoidal sinusitis and mastoiditis. Thrombosis of cerebral sinuses and jugular branches were no more appreciable. Based on the clinical amelioration (significant decrease of frontal headache and diplopia) and the consistent imaging improvement, surgical drainage of sphenoidal sinus was not performed and the patient was discharged after 21 days with indication to switch to Ciprofloxacin 500 $\mathrm{mg} / \mathrm{die}$ for the next 10 days and to start anticoagulation with warfarin. Informed consent was obtained from the patient and the study checked for ethics.

LS and septic CVT can associate because both share identical etiological and physiopathological mechanisms (1,2,5-7). Sometimes, CVT may represent intracranial extension of jugular vein thrombosis. Signs

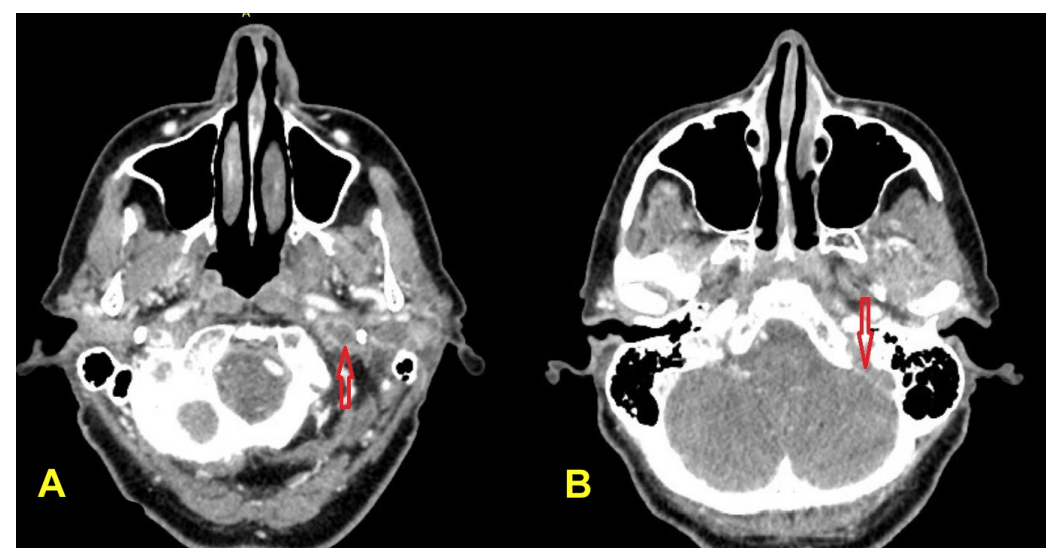

Figure 1. Initial CT scan of the head enhanced with contrast medium. Thrombosis of the jugular left vein (A) and filing defect of the left sigmoid sinus (B).

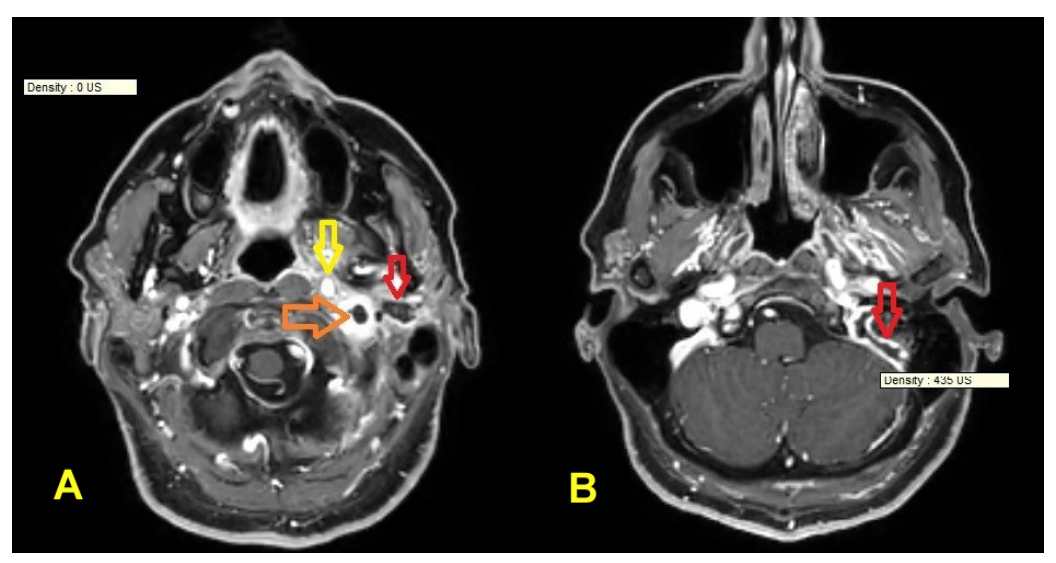

Figure 2. T1 W sequence with gadolinium of transverse head section at the level of pharynx. (A) Filling defect indicating thrombosis at the origin of the left internal jugular vein with hyperintense signal of the surrounding tissue suggestive of inflammatory imbibition (orange arrow). Pseudo-abscess mass diagnosed as parotid adenoma following needle- aspiration (red arrow). Inflammatory solid tissue imprinting the left wall of pharynx (yellow arrow), (B) Filing defect indicating thrombosis of the left sigmoid sinus (red arrow). 
and symptoms of intracranial hypertension such as headache, decreased visual acuity, papilledema and bilateral sixth cranial nerve palsy may be indicative of a CVT condition (8).

In the literature, case reports describing LS complicated with CVT are few $(5,9,10)$. Therapeutic protocol include treatment with antibiotics combined with local surgical drainage and removal of the infected site, with poor outcome in half of the cases and side effects spanning from mild hearing impairment (specially in children) to iatrogenic facial palsy (10).

Neck MRI in our patient initially suggested the presence of an abscess in the context of parotid gland, so making possible a surgical drainage. However, this was a confounding detection. In fact appropriate needleaspiration clarified the adenomatous origin of the mass. Inflammatory imbibition of the surrounding tissues of the internal jugular vein as well as the presence of left mastoiditis and sphenoidal sinusitis were nevertheless clearly evident and could be the causative triggers of septic thrombophlebitis. Currently, precise management protocols of LS with CVT complication do not exist. Choice of antibiotics still follows empiric criteria principally based on expert knowledge (3) and were immediately started in our patient as well as subcutaneous enoxaparin b.i.d. Enoxaparin was chosen as it was considered the most manageable anticoagulation therapy while waiting for a drainage decision. When discharged, the patient was advised to bridge enoxaparin to warfarin. A clinical followup three months later showed that she had completely recovered. As in previous reports (5), this is another evidence suggesting that surgical drainage is not a necessary step in all cases of LS complicated with CVT and that a conservative approach may avoid fearsome complications.

\section{Funding: None.}

Conflict of Interest: The authors have no conflicts of interest to disclose.

\section{References}

1. Lemierre A. On certain septicaemias due to anaerobic organisms. Lancet 1936; 227:701-703.

2. Hagelskjaer Kristensen L, Prag J. Lemierre's syndrome and other disseminated Fusobacterium necrophorum infections in Denmark: a prospective epidemiological and clinical survey. Eur J Clin Microbiol Infect Dis. 2008; 27:779-789.

3. Johannesen KM, Bodtger U. Lemierre's syndrome: current perspectives on diagnosis and management. Infect Drug Resist. 2016; 9:221-227.

4. Hagelskjaer Kristensen L, Prag J. Human necrobacillosis, with emphasis on Lemierre's syndrome. Clin Infect Dis. 2000; 31:524-532.

5. Baltasar-Corral J, Martín-Rojas RM, Parra-Virto A, Galeano-Valle F, Del-Valle-Diéguez M, Del-Toro-Cervera J, Demelo-Rodríguez P. Torcular herophili and lateral sinus thrombosis: An atypical presentation of Lemièrre's syndrome. Intractable Rare Dis Res. 2019; 8:206-209.

6. Wang L, Duan J, Bian T, Meng R, Wu L, Zhang Z, Zhang X, Wang C, Ji X. Inflammation is correlated with severity and outcome of cerebral venous thrombosis. J Neuroinflammation. 2018; 15:329.

7. Fleet J, Birns J, Bhalla A. Cerebral venous thrombosis in adults. J Neurol Disord Stroke. 2014; 2: 1033.

8. Ferro JM, Canhao P, Aguiar de Sousa D. Cerebral venous thrombosis. Presse Med. 2016; 45:e429-e450.

9. Villamar MF, Lee JD. Cerebral venous sinus thrombosis secondary to otomastoiditis. Postgrad Med J. 2017; 93:569.

10. Bales CB, Sobol S, Wetmore R, Elden LM. Lateral sinus thrombosis as a complication of otitis media: 10-year experience at the children's hospital of Philadelphia. Pediatrics. 2009; 123:709-713.

Received November 16, 2021; Revised January 23, 2022; Accepted February 6, 2022.

*Address correspondence to:

Maurizio Giorelli, Operative Unit of Neurology, "Dimiccoli" General Hospital, Viale Ippocrate 11, Barletta 76121, Italy. E-mail: maurizio.giorelli@aslbat.it

Released online in J-STAGE as advance publication February 10, 2022. 